\title{
Discharge planning and home follow up by advanced practice nurses reduced hospital readmissions of elderly patients
}

Naylor MD, Brooten D, Campbell R, et al. Comprehensive discharge planning and home follow-up of hospitalized elders. A randomized clinical trial.JAMA 1999 Feb 17;281:613-20.

\section{Question}

In elderly people admitted to hospital, does a discharge planning and home follow up protocol implemented by advanced practice nurses (APNs) improve patient outcomes and reduce healthcare costs?

\section{Design}

Randomised (concealed), single blind (outcome assessor) controlled trial with follow up at 24 weeks.

\section{Setting}

2 university affiliated hospitals in Philadelphia, Pennsylvania, USA.

\section{Patients}

363 patients $\geq 65$ years of age (mean age 75 y, $50 \%$ men, $55 \%$ white) who were admitted from home with 1 of congestive heart failure, angina, myocardial infarction, respiratory tract infection, coronary artery bypass graft, cardiac valve replacement, major small and large bowel procedure, or lower extremity orthopaedic procedure. All patients had $\geq 1$ risk factor for poor discharge outcomes ( $\geq 80$ years of age; inadequate support system; multiple, chronic health problems; history of depression; moderate to severe functional impairment; multiple hospital admissions during previous $6 \mathrm{mo}$; hospital admission in previous $30 \mathrm{~d}$; fair or poor self rated health; or history of non-adherence to treatment). 262 patients (72\%) completed the study.

\section{Intervention}

177 patients were allocated to the APN intervention, which extended from admission to 4 weeks after discharge. 5 gerontological APNs implemented a standardised discharge planning and home follow up protocol that included APN visits during hospital stay, home visits after discharge, telephone availability, and weekly telephone contact. 186 patients were allocated to the control group and received routine discharge planning, including standard home care if referred.

\section{Main outcome measures}

Outcomes included readmissions (cumulative hospital days, mean length of stay), time to first readmission, estimated cost of health services after discharge (based on standardised Medicare reimbursements), patient functional status, depression, and satisfaction.

\section{Main results}

Readmission results were based on all allocated patients. At 24 weeks, patients in the APN group were less likely to be readmitted at least once (table) and they had a longer time to first readmission $(\mathrm{p}<0.001)$; readmitted patients in the APN group had shorter stays (mean length of stay $7.5 v 11.0 \mathrm{~d}, \mathrm{p}<0.001$ ). They had fewer days in hospital (mean $1.53 v 4.09 \mathrm{~d}, \mathrm{p}<0.001$ ) and lower costs (US\$3630 v \$6661/patient, $\mathrm{p}<0.001$ ). The groups did not differ for functional status, depression, or patient satisfaction.

\section{Conclusion}

Among elderly inpatients at risk for hospital readmission, discharge planning and home follow up by an advanced practice gerontological nurse reduced hospital readmissions, increased length of time from discharge to readmission, and reduced healthcare costs compared with usual care.

Advance practice nurse (APN) discharge and follow up protocol v usual care at 24 weeks*

\begin{tabular}{lllll}
\hline Outcome & $\begin{array}{l}\text { APN } \\
\text { protocol }\end{array}$ & Control & RRR (95\% CI) & NNT (CI) \\
\hline$\geq 1$ readmission & $20.3 \%$ & $37.1 \%$ & $45.2 \%(22.9$ to 61.3$)$ & $6(4$ to 14$)$ \\
\hline
\end{tabular}

*Abbreviations defined in glossary; RRR, NNT, and CI calculated from data inarticle.

\section{Source of funding: National Institute for Nursing Research.}

For correspondence: Dr M D Naylor, University of Pennsylvania, School of Nursing, 420 Guardian Drive, Philadelphia, PA 19104, USA. Fax +1 2155736659.

A modified version of this abstract appears in ACP Journal Club.
This study by Naylor $e t$ al adds to the growing body of research showing the positive effects of care delivered by APNs. The results show the human and financial value of individualised discharge planning based on a biopsychosocial model of care as well as attending to the transition between hospital and home, continuity of care, and interdisciplinary collaboration. The elderly patients in the study had one of several diagnoses, demonstrating that this intervention need not be disease specific.

Because the study was done in the US, the results cannot be generalised to other countries that may have different sociodemographic characteristics and reimbursement policies. The entire sample had high mental status examination scores and low depression scores, and therefore the effectiveness of similar interventions delivered to other high risk groups (eg, older adults with cognitive impairment or depression) is unknown. Also, generalisability may be limited because the sample consisted of primarily white, urban dwelling elderly people.The intervention did not affect functional status in the home, a finding that is concerning, but not surprising, and is consistent with the evolving geriatric literature on functional status in high risk elders after discharge from a specialised unit for acute elder care. ${ }^{12}$ Despite lack of improvement in functional status, there were reductions in readmissions. The authors suggest that the APN intervention enhanced the capacity of high risk elderly patients to cope with their multiple medical problems and disabilities. The APNs had master's degrees and were gerontological nurse specialists who, in collaboration with the patient, physician, caregiver, and other team members, designed and implemented an individualised discharge plan within the bounds of the protocol. This study shows the value of continuous, individualised care during transitions from acute to post-acute care.

Maureen C Shaw, RN, MN Clinical Nurse Specialist, Acute Gerontology Vancouver Hospital Eं Health Sciences Centre Vancouver, British Columbia, Canada

1 Covinsky KE, Palmer RM, Kresevic DM, et al.Jt Comm J Qual Improv 1998;24:63-76.

2 Palmer RM, Counsell S, Landefeld CS. Clin Geriatr Med 1998;14:831-49. 